\title{
La determinación de la aptitud a la molienda y del cosumo específico de energía según el ensayo de Bond para la molienda de minerales y rocas
}

\author{
Dipl. Ing. H. D. WASMUTH, Clausthal-zellerfold \\ Aufbereitungs-Technik, n 6, junio 1969, págs. $284-289$
}

\section{resumem}

Durante las dos últimas décadas se han elaborado varios métodos de laboratorio para determinar la aptitud a la molienda y el consumo específico de energía. En lo que concierne a la molienda de minerales en molinos de bolas se utiliza frecuentemente, por lo menos en los Estados Unidos, el ensayo de Bond, pudiendo procederse a la explotación de los resultados con el apoyo de diversas relaciones empíricas.

En el presente trabajo se describen sumariamente los distintos métodos. Se discuten los resultadoș de ensayos de molienda, según Bond, realizados en diversos minerales y rocas y se los compara con los datos de instalaciones de molienda industriales.

\section{INTRODUCCION}

Como es sabido, para la trituración de minerales y rocas en molinos hay que gastar grandes cantidades en energía, de las cuales solamente una parte muy pequeña, menos del $1 \%$, se consume como trabajo útil que conduce a un aumento de la superficie del material, mientras que la mayor parte, más del $99 \%$, se pierde, sobre todo en forma de calor y de cambios en el régimen de trabajo mecánico. El consumo total de energía para la molienda de un material en un molino tubular se influye, según Rose, (1), por muchísimos parámetros que, por una parte, vienen dados por las propiedades específicas del material a moler y, por otra, por los datos de servicio y la construcción del molino, así como también por el tamaño y el tipo de los cuerpos molturadores.

Años atrás se han propuesto varios métodos para determinar la aptitud y el consumo de energía en la molienda de minerales y rocas en molinos de bolas, los cuales permiten averiguar, mediante ensayos de laboratorio relativamente sencillos, la aptitud y el gasto de energía para su molienda desde un tamaño de grano predeterminado hasta un tamaño del producto molido, sin tener que realizar ensayos minuciosos en molinos de laboratorio o costosos ensayos de molienda en molinos industriales. 


\section{METODOS DE LABORATORIO PARA DETERMINAR LA APTITUD A LA MOLIENDA Y EL CONSUMO ESPECIFICO DE ENERGIA}

Los métodos en uso se pueden dividir, fundamentalmente, en tres grupos:

a) métodos basados en la "ley de trituración" de Bond;

b) métodos que, independientemente de Bond, trabajan también con molinos de bolas de laboratorio como aparatos de análisis;

c) métodos que trabajan con otras instalaciones de molienda como aparatos de análisis.

\subsection{Métodos basados en la teoría de la trituración de Bond}

Los métodos de ensayo de la aptitud a la molienda del primer grupo se basan en la tercera teoría de trituración derivada empíricamente por Bond, (2). Esta teoría parie de la base de que la energía consumida para la trituración es proporcional a la longitud de las grietas que se producen en la trituración; y en el supuesto de un material homogéneo establece la siguiente relación empírica para el consumo de fuerza $W_{B}$ en la trituración de una granulometría discrecional de entrada a una finura final dada:

$$
\mathrm{W}_{\mathrm{B}}=\frac{10 \mathrm{Wi}_{\mathrm{B}}}{\sqrt{\mathrm{P}}}-\frac{10 \mathrm{Wi}_{\mathrm{B}}}{\sqrt{\mathrm{F}}} \quad(\mathrm{kWh} / \mathrm{t})
$$

o introduciendo la relación de trituración $\mathrm{R}=\mathrm{F} / \mathrm{P}$ :

$$
\mathrm{W}_{\mathrm{B}}=\mathrm{Wi}_{\mathrm{B}}\left(1-\frac{1}{\mathrm{R}^{0,5}}\right)\left(\frac{100}{\mathrm{P}}\right)^{0,5} \quad(\mathrm{kWh} / \mathrm{t})
$$

Para ello se define la constante del material Wi (índice de trabajo) como la demanda energética (en $\mathrm{kWh} / \mathrm{t}$ ) que es necesaria para la trituración de un material de tamaño de grano indefinido hasta llegar a un $80 \%$ menor de $100 \mu ; \mathrm{F}$ y $\mathrm{P}$ son los valorés de $\mathrm{D}_{80}$ de alimentación y producto molido, es decir, aquellas aperturas de malla (en $\mu$ ) en las cuales el $80 \%$ de peso de la alimentación (F) o del producto acabado (P) llegan a transpasar el tamiz.

En el supuesto de material homogéneo, el índice de trabajo Wi representa una constante del material. Con materiales heterogéneos, es decir, en casi todos los minerales y rocas, los índices de trabajo dependen de la finura final de la molienda y de otros factores.

En consecuencia, Holmes, (3), propone considerar como variable el exponente de trituración que Bond indica con 0,5 , con el fin de que el indice de trabajo Wi conserve el carácter de una constante real del material. La ecuación de Bond [2] la transforma en:

$$
\mathrm{W}_{\mathrm{H}}=\mathrm{Wi}_{\mathrm{H}}\left(1-\frac{1}{\mathrm{R}^{\mathrm{r}}}\right)\left(\frac{100}{\mathrm{P}}\right)^{\mathrm{r}} \quad(\mathrm{kWh} / \mathrm{t})
$$

En ensayos de laboratorio encontró para el exponente $r$ valores que oscilan de 0,2 a 1,4.

El ensayo de molienda de Bond, (4), para determinar el índice de trabajo Wi, se realiza en un molino de bolas de $305 \times 305 \mathrm{~mm}$ con paredes lisas y un número de revoluciones de 70 r.p.m. igual al $91 \%$ de $\mathrm{n}_{\text {crit }}$ (velocidad crítica) y con una carga de bolas definida 
exactamente, en número y tamaño, de $20,125 \mathrm{~kg}$ en seco, añadidas, poco a poco, hasta alcanzar un estado de molienda uniforme, como se describe con más detalle en el apartado 3.1. El índice de trabajo Wi hallado da el consumo energético específico para un molino de bolas, de $2,45 \times 2,45 \mathrm{~m}$, que trabaja en húmedo en condiciones exactamente determinadas en lo que se refiere al número de revoluciones, carga del molino, altura de la carga, etc.

Un' método similar para la determinación de la capacidad de trituración de minerales lo dan Perow-Brand con arreglo a 'los análisis de Schirokinski, (5). Los ensayos se efectuaron según el método indicado por Bond con un molino de iguales dimensiones, un número de revoluciones ligeramente modificado (68,5 r.p.m.), una carga de bolas alterada en su composición y una carga de $300 \%$. Como medida de la aptitud a la trituración se considera el rendimiento específico (en $\mathrm{kg} / \mathrm{h}$ ) por unidad del volumen del molino con una trituración hasta diversas finuras finales.

\subsection{Otros métodos que utilizan molinos de bolas de laboratorio}

A los ensayos de laboratorio del grupo segundo pertenece el método desarrollado por Mittag, (6), para determinar la resistencia específica de molienda y el consumo de energía específico en un molino de laboratorio, del cual se toman, a intervalos regulares, muestras del producto molido que se analizan en cuanto a su grado de finura. El consumo energético específico A se cálcula:

$$
\mathrm{A}=\frac{\mathrm{M} \cdot \mathrm{n} \cdot \mathrm{z}}{58.500 \cdot \mathrm{Q}} \quad(\mathrm{kWh} / \mathrm{t})
$$

en la cual:

M significa el momento de giro del molino (en $\mathrm{m} \cdot \mathrm{kp}$ );

n, el número de revoluciones del molino (en r.p.m.);

Q, la cantidad del producto a moler (en t).

En el supuesto de que el molino de laboratorio utilizado tenga un diámetro de más de $800 \mathrm{~mm}$, para la traslación de los valores de consumo energético hallados en el ensayo de laboratorio a los molinos industriales se emplea la relación

$$
\mathrm{A}=\frac{\mathrm{A}_{\mathrm{L}}}{\mathrm{Q}_{\mathrm{L}}}=\frac{\mathrm{A}_{\mathrm{B}}}{\mathrm{Q}_{\mathrm{B}}} \quad(\mathrm{kWh} / \mathrm{t}),
$$

siempre que el molino de laboratorio trabaje en las mismas condiciones que el molino industrial.

En esta ecuación:

$A_{L}$ es la energía absorbida por el molino de laboratorio (en $\mathrm{kW}$ );

$\mathrm{Q}_{\mathrm{L}}$, la producción del molino del laboratorio (en $\mathrm{t} / \mathrm{h}$ );

$A_{B}$, la energía absorbida por el molino industrial (en $\mathrm{kW}$ );

$\mathrm{Q}_{\mathrm{B}}$, la produción del molino industrial (en $\mathrm{t} / \mathrm{h}$ ).

También la Denver Equipment Co., (7), ha desarrollado, en su división de ensayo de mi- 
nerales, un método de laboratorio para determinar la aptitud a la molienda. En un molino de laboratorio de $305 \times 127 \mathrm{~mm}$, con una carga de bolas de $18,15 \mathrm{~kg}$ y un número de revoluciones de 54 r.p.m., se muelen, durante 3 minutos, $2 \mathrm{~kg}$ de mineral previamente triturado a menos de $1,6 \mathrm{~mm}$; a continuación, se tamiza con abertura de malla de $74 \mu$. La fracción de menos de $74 \mu$ se sustituye por material crudo (alimentación primaria) y se repite la molienda durante un tiempo igual. Estos pasos individuales se efectúan hasta que un $80 \%$ de la alimentación primaria se haya pulverizado a menos de $74 \mu$. La producción de menos de $74 \mu$ después de cada intervalo se introduce en el diagrama representado en la figura 1 , y la resistencia relativa a la molienda del material analizado se determina por comparación con las curvas standard dibujadas. Un método gráfico de valoración facilita determinar el tamaño correspondiente del molino para la molienda industrial de una cantidad determinada del material analizado a una finura final discrecional.

\subsection{Otros métodos de laboratorio}

Además de los molinos de bolas de laboratorio, para determinar la aptitud a la molienda de ciertos materiales, sobre todo de carbón y cemento, se emplean también molinos de anillos y de rodillos, como desarrollaron, entre otros, Hardgrove y Zeisel. Ambos aparatos trabajan fundamentalmente según el mismo principio.

El aparato de análisis de Hardgrove, (8), se compone de un plato de molienda, en forma de anillo, en el que ocho bolas molturadoras son impulsadas por un anillo giratorio de contacto. En el ensayo de molturabilidad se emplean $50 \mathrm{~g}$ de material de granulometría comprendida entre 1,2 y $0,6 \mathrm{~mm}$. Se tritura

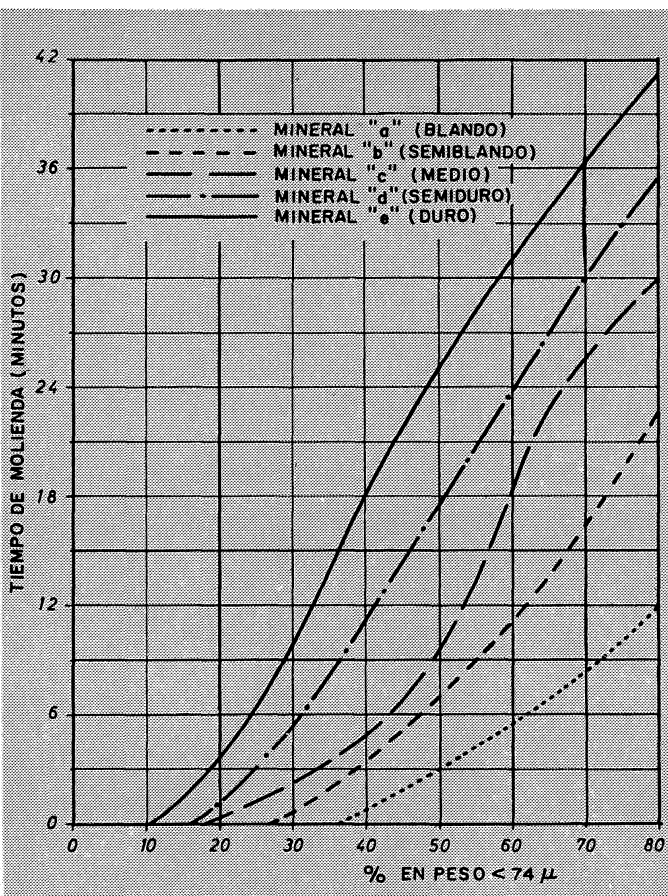

Fig. 1.-Dlagrama para determinar la resistencla relativa a la molienda, segtin Denver Equipment. a 60 r.p.m. y se tamiza con $74 \mu$. De la cantidad $\mathrm{m}_{\mathrm{H}}$, que se produce a menos de $74 \mu$, se calcula el índice de molturabilidad $\mathrm{G}_{\mathrm{H}}$ según la fórmula empírica:

$$
\mathrm{G}_{\mathrm{H}}=13+6,93 \cdot \mathrm{m}_{\mathrm{H}} \text {. }
$$

Otro método para determinar la aptitud a la molienda de un producto en el laboratorio lo describen Lehmann y Haese, (9); se trata del comprobador de molturabilidad desarrollado por Zeisel para determinar la resistencia a la molienda, el cual indica la aptitud a la molienda (en $\mathrm{m} \cdot \mathrm{kp} / \mathrm{g}$ ) para el aumento específico de superficie $\left(\mathrm{en} \mathrm{cm}^{2} / \mathrm{g}\right.$ ). En este aparato se echan $30 \mathrm{~g}$ de material de una determinada granulometría $(1,0-0,75$ milímetros) a un estrecho plato molturador de forma circular cargado con ocho bolas de 25,4 $\mathrm{mm}$ de diámetro. Sobre las bolas se comprime con $25 \mathrm{~kg}$ de carga un anillo molturador que gira a 200 r.p.m. La inclinación del plato molturador se transforma a través del contacto deslizante de un potenciómetro en corriente eléctrica y representa una medida de 
la resistencia a la molienda. Por una magnitud de contraste, que indica en $\mathrm{m} \cdot \mathrm{kp}$ la reducción de la energía eléctrica averiguada en un mecanismo contador, se determina la aptitud a la molienda. La médición del aumento de superficie se realiza según Blaine.

El comprobador de molturabilidad sólo se ha aplicado en la industria del cemento. Los resultados obtenidos con este aparato deben concordar con los resultados industriales de la molienda del cemento, aproximadamente en la relación $1: 2$.

\section{ENSAYOS DE LABORATORIO SEGUN BOND}

De los métodos descritos para la determinación en el laboratorio de la molturabilidad y el consumo energético específico, el ensayo de aptitud a la molienda desarrollado por Bond ha alcanzado la máxima importancia - por lo menos en Norteamérica- en el dimensionado y comprobación de instalaciones de molienda de minerales. Por lo tanto, se efectuaron ensayos de laboratorio en el molino de Bond con diferentes minerales y rocas, determinando la aptitud y ei consumo energético en la molienda de estos materiales. Para comprobar la exactitud de los resultados obtenidos, se compararon con los valores conseguidos en la molturación industrial.

El ensayo de Bond presenta algunas ventajas frente a los otros métodos de laboratorio. En contraposición al ensayo de Hardgrove y para el comprobador de aptitud a la molienda tipo industrial de la arcilla, el proceso de trituración en el molino de Bond concuerda con el proceso de molienda en el molino industrial en su mayor parte, ya que en ambos molinos se producen los mismos tipos de esfuerzos. Frente al método de laboratorio para determinar la resistencia específica a la molienda según Mittág, el ensayo de Bond tiene la ventaja de que puede utilizarse un molino considerablemente menor, por lo que se reducen notablemente las cantidades que hay que molturar.

Además, en el ensayo de Bond existen condiciones exactamente definidas en cuanto a cargas de bolas, etc., en contraposición al método aplicado por Denver Equipment. En este último método hay que variar la carga de bolas hasta que las curvas del tanto por ciento de peso de menos de $74 \mu$ muestren, en función del tiempo de molienda, aproximadamente la misma inclinación que las curvas-patrón (fig. 1). Para ello se han efectuado algunas series de ensayos con diferentes materiales. Las curvas obtenidas en estos ensayos eran de un recorrido mucho más aplanado que el que exigían las curvas-patrón. Por ejemplo, la curva de un mineral, que según la experiencia era muy malo de molturar al aumentar el tiempo de la molienda, cortaba todas las curvas standard de minerales desde "e" hasta " $b$ ". Por consiguiente, no fue posible clasificar este mineral en ninguna de las cinco categorías.

Como lo demuestran los estudios hechos en el laboratorio Warren Spring, (10), el ensayo de Bond sirve también para determinar el índice de trabajo Wi según Holmes y los exponentes de trituración $\mathrm{r}$ introducidos en él como variables, si en los ensayos de laboratorio se realiza una molienda a diversas finuras finales.

\subsection{Realización del ensayo de Bond}

El mineral a ensayar se tritura previamente y con cuidado a menós de $3,3 \mathrm{~mm}$. La alimentación al molino es de $700 \mathrm{~cm}^{3}$ de este material. Se tritura en seco con una carga circulante supuesta de $250 \%$ a modo de cargas, a la finura final deseada del producto a mo- 
ler. Para ello, la molienda se realiza escalonadamente hasta conseguir un régimen de molienda uniforme, en la siguiente forma:

En el primer ciclo de molienda se ajusta el número de revoluciones del molino según valores prácticos de los que ya se dispone para materias similares. Una vez concluida la molienda, todo el producto molido se tamiza con el paso de tamiz $\mathrm{P}_{\mathrm{i}}$ previamente elegido. La granulometría superior, juntamente con una parte de la cantidad de material de alimentación que corresponde a la parte de granulometría fina separada, vuelve a echarse al molino y se la moltura de nuevo. El nuevo número de revoluciones se cálcula para la carga circulante dada de $250 \%$ a través del coeficiente de aptitud a la molienda G (grindability), que indica la cantidad de granulometría inferior producida de nuevo por cada revolución del molino. Este método, que en la tabla 1 se representa para la molienda de tierra ocre de menos de $0,2 \mathrm{~mm}$, se repite hasta que el coeficiente de molturabilidad G permanezca constante a través de tres períodos de molienda. El producto fino de los tres últimos ciclos de molienda - con el que se ha hecho un todo uno- y el de la alimentación primaria del molino se someten a un análisis granulométrico.

Los análisis del tamizado se registran en una escala doblemente logarítmica propuesta por Bond, (11), según la figura 2, en la que se determinan los valores de $\mathrm{D}_{80}$ de alimentación $(\mathrm{F})$ y producto molido $(\mathrm{P})$.

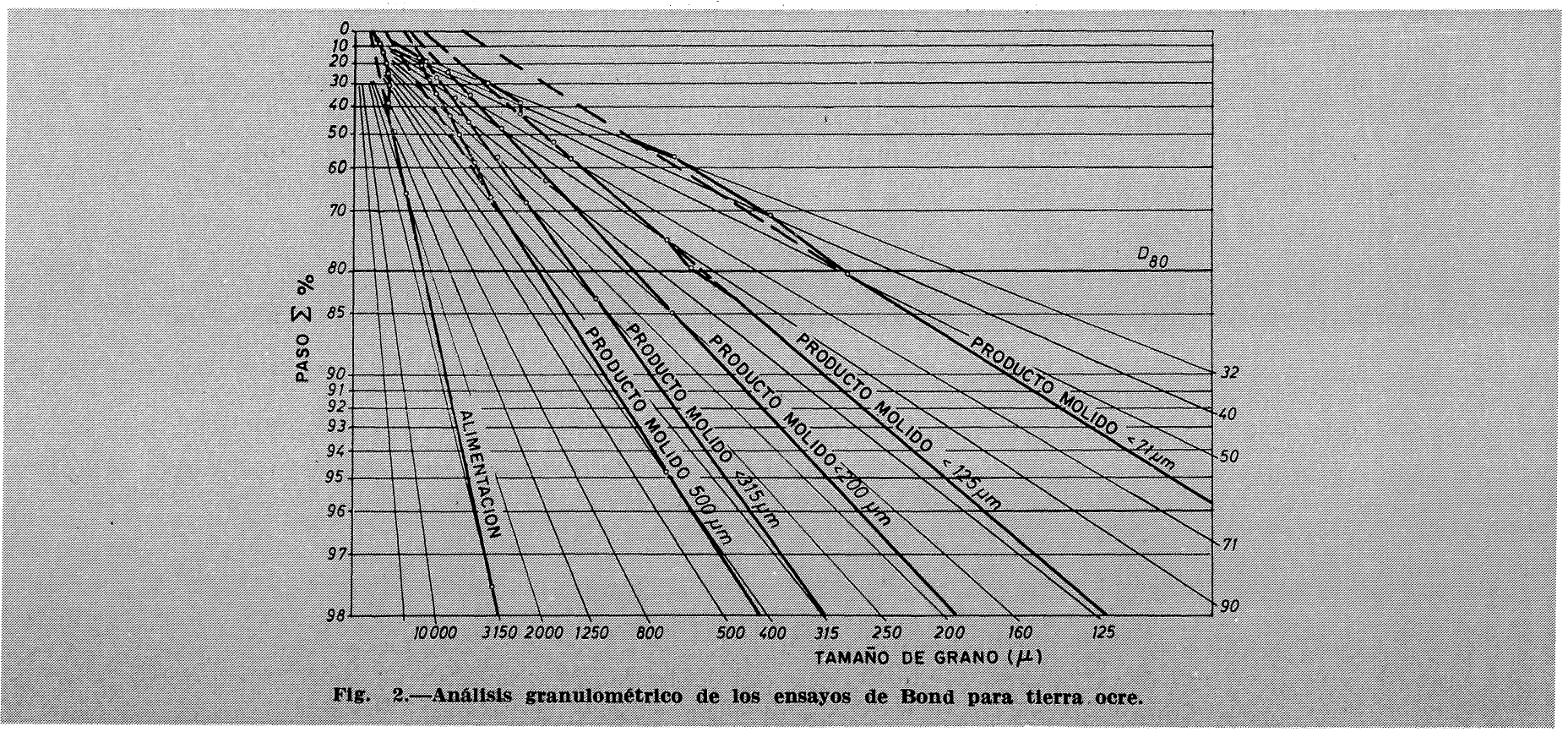

Los análisis de aptitud a la molienda, según Bond, se efectuaron en los siguientes minerales y rocas:

- mineral rico Rammelsberg,

- mineral en betas Rammelsberg,

- mineral Pb-zn, Lüderich,

-- mineral Pb-Maubach,

- mineral de espato-flúor Finstergrund,

- piedra caliza (Iberg, Harz),

- grauwaca (Harz), 
con tamizado intermedio en los tamaños de grano de referencia (finuras de molienda); $P_{i}=500,315,200,125$ y $71 \mu$. Los resultados de los valores obtenidos para el coeficiente de aptitud a la molienda $\mathrm{G}(\mathrm{g} / \mathrm{U})$ y el correspondiente tamaño de grano del producto molido $P$ se ven en la figura 3 y en la tabla 2.

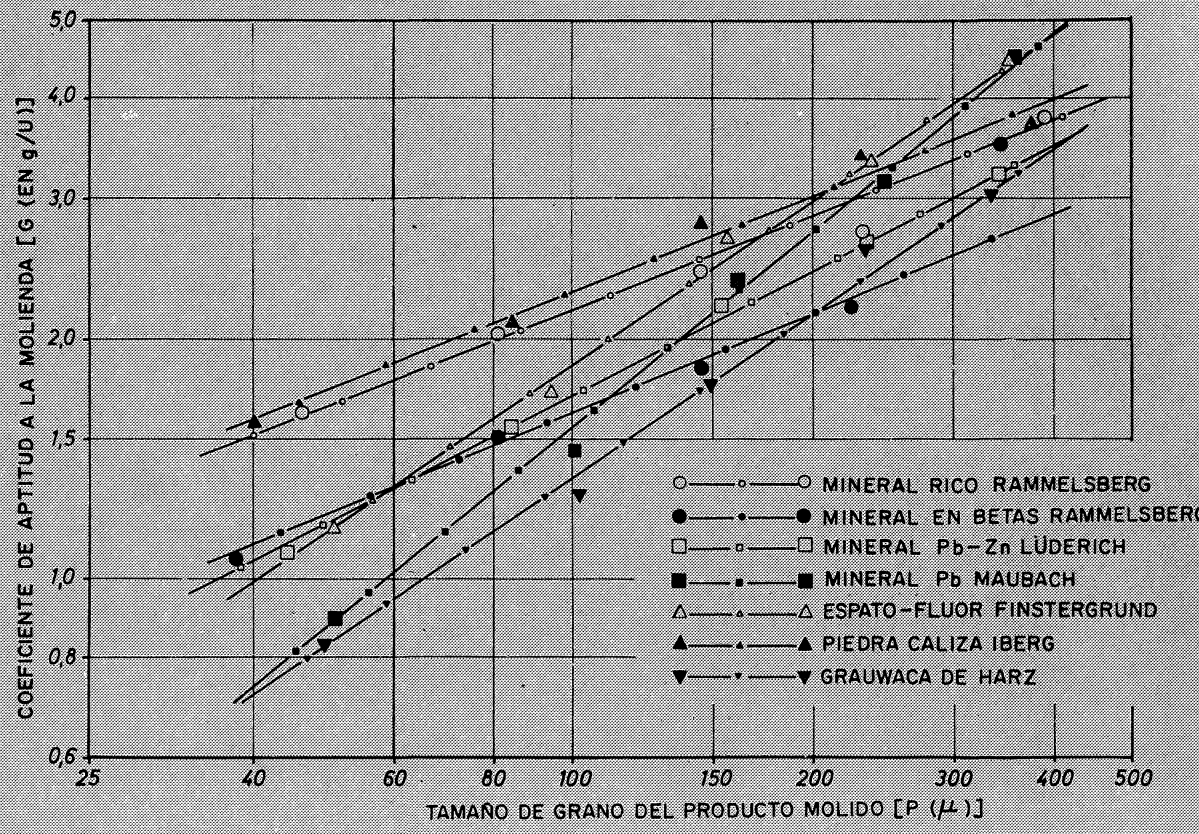

Fig. 3.-Coeficiente de aptitud a la molienda G y tamaño de grano del producto molido $P$ de los ensayos de Bond.

\subsection{Valoración del ensayo de Bond}

La valoración de los ensayos de molienda realizados se hizo, una vez, de acuerdo con las dos fórmulas indicadas por Bond [(2) y (12)]:

$$
\mathrm{Wi}_{\mathrm{B}}=\frac{4,45}{\mathrm{P}_{\mathrm{i}}^{0,23} \cdot \mathrm{G}^{0,82}\left(\mathrm{P}^{0,5}-\mathrm{F}^{0,5}\right)} \quad(\mathrm{kWh} / \mathrm{t})
$$

$\mathrm{y}$

$$
\mathrm{Wi}_{\mathrm{B}}=\frac{16}{\mathrm{G}^{0,82}}\left(\frac{\mathrm{P}_{\mathrm{i}}}{100}\right)^{0,5} \quad(\mathrm{kWh} / \mathrm{t}) .
$$

En otra ocasión, los datos de los ensayos de molienda se valoraron según la relación desarrollada por Holmes (3):

$$
\mathrm{Wi}_{\mathrm{H}}=\frac{0,8 \cdot \mathrm{A}}{\mathrm{Gq}}\left(\frac{\mathrm{P}}{100}\right)^{\mathrm{r}} \quad(\mathrm{kWh} / \mathrm{t}) .
$$

La determinación de las constantes adimensionales A, q y r que en la tabla 3 están indicadas para los diversos materiales, se realizó por la triple aplicación del método de aproximación descrito por Holmes y Warren Spring Laboratory. 
T A B L A 1

Protocolo de ensayo de una prueba de molienda en el molino de Bond

Objeto del ensayo: molienda a menos de $0,2 \mathrm{~mm}$.

Producto a moler: grauwaca.

Peso de la carga: $1.195 \mathrm{~g}$.

Proporción $-0,2 \mathrm{~mm}$ en la alimentación: $20,4 \%$ en peso.

Proporción de la carga circulante: $250 \%$.

\begin{tabular}{|c|c|c|c|c|c|}
\hline \multirow{2}{*}{ Período } & \multirow{2}{*}{$\begin{array}{l}\text { Número de } \\
\text { revoluciones }\end{array}$} & \multicolumn{3}{|c|}{ Producción-0,2 mm } & \multirow{2}{*}{$\begin{array}{c}\text { Coeficiente de } \\
\text { molturabilidad } \\
\text { G } \\
(\mathrm{g} / \mathrm{C})\end{array}$} \\
\hline & & $\begin{array}{c}\text { En el producto } \\
\text { molido } \\
(\mathrm{g})\end{array}$ & $\begin{array}{c}\text { En la alimentación } \\
\text { (g) }\end{array}$ & $\begin{array}{c}\text { Producido de nuevo } \\
\text { (g) }\end{array}$ & \\
\hline 1 & 105 & 367,5 & 244,0 & 123,5 & 1,17 \\
\hline 2 & 234 & 430,0 & 77,0 & 353,0 & 1,51 \\
\hline 3 & 169 & 364,0 & 87,5 & 276,5 & 1,64 \\
\hline 4 & 163 & 358,0 & 76,0 & 282,0 & 1,73 \\
\hline 5 & 155 & 347,5 & 73,0 & 274,5 & 1,77 \\
\hline 6 & 153 & 338,0 & 71,0 & 267,0 & 1,75 \\
\hline 7 & 155 & 341,0 & 69,0 & 272,0 & 1,76 \\
\hline 8 & 155 & 343,0 & 69,5 & 273,5 & 1,76 \\
\hline
\end{tabular}


T A B L A 2

Resultados de los ensayos de molienda en el laboratorio en el molino de Bond

\begin{tabular}{|c|c|c|c|c|c|c|}
\hline M a t erial & $\begin{array}{c}\text { Finura de } \\
\text { molido } \\
\mathbf{P}_{\mathbf{i}} \\
(\mu)\end{array}$ & $\begin{array}{c}\text { Tamaño de } \\
\text { grano del } \\
\text { producto } \\
\text { molido } \\
\text { P } \\
(\mu)\end{array}$ & $\begin{array}{l}\text { Coeficiente } \\
\text { de molturabi- } \\
\text { lidad } \\
\qquad \underset{G}{(g / U)}\end{array}$ & $\begin{array}{c}\text { Indice de tra- } \\
\text { bajo Bond se- } \\
\text { gún }[8] \\
\quad W i_{B} \\
\quad(k W h / t)\end{array}$ & $\begin{array}{l}\text { Indice de tra- } \\
\text { bajo Bond se- } \\
\text { gún }[9] \\
\quad W i_{\mathrm{B}} \\
(\mathrm{kWh} / \mathrm{t})\end{array}$ & $\begin{array}{c}\text { Indice de tra- } \\
\text { bajo Holmes } \\
\text { según }[10] \\
\quad \mathbf{W i}_{\mathrm{H}} \\
\quad(\mathrm{kWh} / \mathrm{t})\end{array}$ \\
\hline (1) & (2) & (3) & (4) & (5) & (6) & (i) \\
\hline \multirow[t]{2}{*}{$\begin{array}{l}\text { Mineral rico } \\
\text { Rammelsberg }\end{array}$} & $\begin{array}{r}500 \\
315 \\
200 \\
125 \\
71\end{array}$ & $\begin{array}{r}390 \\
230 \\
145 \\
81 \\
46 \\
\end{array}$ & $\begin{array}{l}3,79 \\
2,73 \\
2,43 \\
2,03 \\
1,62\end{array}$ & $\begin{array}{r}13,70 \\
13,10 \\
11,95 \\
10,20 \\
9,90 \\
\end{array}$ & $\begin{array}{r}13,20 \\
13,00 \\
12,30 \\
11,00 \\
9,95\end{array}$ & $\begin{array}{l}13,05 \\
14,15 \\
13,55 \\
14,05 \\
12,95 \\
\end{array}$ \\
\hline & Media & & & 11,80 & 11,90 & 13,55 \\
\hline \multirow[t]{2}{*}{$\begin{array}{l}\text { Mineral en betas } \\
\text { Rammelsberg }\end{array}$} & $\begin{array}{r}500 \\
315 \\
200 \\
125 \\
71 \\
\end{array}$ & $\begin{array}{r}343 \\
223 \\
145 \\
81 \\
38 \\
\end{array}$ & $\begin{array}{l}3,50 \\
2,20 \\
1,84 \\
1,51 \\
1,06\end{array}$ & $\begin{array}{l}13,00 \\
15,15 \\
14,30 \\
12,90 \\
12,35\end{array}$ & $\begin{array}{l}14,10 \\
16,35 \\
15,10 \\
14,05 \\
14,40 \\
\end{array}$ & $\begin{array}{l}12,75 \\
15,65 \\
15,65 \\
15,20 \\
15,75 \\
\end{array}$ \\
\hline & Media & & & 13,55 & 14,80 & 15,00 \\
\hline \multirow[t]{2}{*}{$\begin{array}{l}\text { Mineral } \mathrm{Pb}-\mathrm{Zn} \\
\text { Lüderich }\end{array}$} & $\begin{array}{r}500 \\
315 \\
200 \\
125 \\
71 \\
\end{array}$ & $\begin{array}{c}343 \\
232 \\
152 \\
84 \\
44 \\
\end{array}$ & $\begin{array}{l}3,21 \\
2,64 \\
2,20 \\
1,55 \\
1,08\end{array}$ & $\begin{array}{l}13,65 \\
14,50 \\
12,60 \\
12,80 \\
13,30 \\
\end{array}$ & $\begin{array}{l}15,15 \\
14,05 \\
12,55 \\
13,75 \\
13,95\end{array}$ & $\begin{array}{l}12,70 \\
12,95 \\
12,40 \\
12,90 \\
13,25 \\
\end{array}$ \\
\hline & Media & & & 13,40 & 13,90 & 12,85 \\
\hline \multirow[t]{2}{*}{$\begin{array}{l}\text { Mineral Pb } \\
\text { Maubach }\end{array}$} & $\begin{array}{r}500 \\
315 \\
200 \\
125 \\
71\end{array}$ & $\begin{array}{r}357 \\
246 \\
161 \\
101 \\
51\end{array}$ & $\begin{array}{l}4,49 \\
3,16 \\
2,36 \\
1,45 \\
0,89\end{array}$ & $\begin{array}{l}10,70 \\
11,85 \\
12,40 \\
15,20 \\
17,30 \\
\end{array}$ & $\begin{array}{l}11,45 \\
12,15 \\
12,30 \\
14,45 \\
16,30 \\
\end{array}$ & $\begin{array}{l}10,55 \\
10,85 \\
10,35 \\
11,45 \\
10,60\end{array}$ \\
\hline & Media & & & 13,50 & 13,35 & 10,75 \\
\hline \multirow[t]{2}{*}{$\begin{array}{l}\text { E'spato-flúor } \\
\text { Finstergrund }\end{array}$} & $\begin{array}{r}500 \\
315 \\
200 \\
125 \\
71 \\
\end{array}$ & $\begin{array}{r}354 \\
237 \\
156 \\
94 \\
51 \\
\end{array}$ & $\begin{array}{l}4,46 \\
3,33 \\
2,66 \\
1,71 \\
1,16 \\
\end{array}$ & $\begin{array}{l}10,90 \\
11,25 \\
11,15 \\
12,70 \\
12,70 \\
\end{array}$ & $\begin{array}{l}11,55 \\
11,65 \\
11,60 \\
12,70 \\
13,15 \\
\end{array}$ & $\begin{array}{r}9,95 \\
10,10 \\
9,60 \\
10,45 \\
10,20 \\
\end{array}$ \\
\hline & Media & & & 11,80 & 12,15 & 10,05 \\
\hline \multirow[t]{2}{*}{$\begin{array}{l}\text { Piedra caliza } \\
\text { Iberg }\end{array}$} & $\begin{array}{r}500 \\
315 \\
200 \\
125 \\
71 \\
\end{array}$ & $\begin{array}{r}375 \\
230 \\
145 \\
84 \\
40 \\
\end{array}$ & $\begin{array}{l}3,71 \\
3,38 \\
2,78 \\
2,09 \\
1,57\end{array}$ & $\begin{array}{r}13,10 \\
11,25 \\
10,25 \\
10,00 \\
9,25 \\
\end{array}$ & $\begin{array}{r}13,45 \\
11,55 \\
10,80 \\
10,75 \\
10,25 \\
\end{array}$ & $\begin{array}{l}13,30 \\
12,35 \\
12,55 \\
12,95 \\
12,90\end{array}$ \\
\hline & Media & & & 10,70 & 11,35 & 12,80 \\
\hline \multirow[t]{2}{*}{$\begin{array}{l}\text { Grauwaca de } \\
\text { Harz }\end{array}$} & $\begin{array}{r}500 \\
315 \\
200 \\
125 \\
71 \\
\end{array}$ & $\begin{array}{r}334 \\
233 \\
149 \\
102 \\
49 \\
\end{array}$ & $\begin{array}{l}3,05 \\
2,61 \\
1,76 \\
1,28 \\
0,83 \\
\end{array}$ & $\begin{array}{l}13,60 \\
13,10 \\
14,60 \\
16,90 \\
17,55\end{array}$ & $\begin{array}{l}15,75 \\
14,20 \\
15,65 \\
16,15 \\
17,25 \\
\end{array}$ & $\begin{array}{l}13,10 \\
12,15 \\
13,15 \\
13,80 \\
13,10\end{array}$ \\
\hline & Media & & & 15,15 & 15,80 & 13,05 \\
\hline
\end{tabular}


T A в L A 3

Constantes para la valoración del ensayo de Bond según Holmes

\begin{tabular}{|c|c|c|c|}
\hline M a t e r la 1 & $\mathbf{A}$ & $\mathbf{q}$ & $\mathbf{r}$ \\
\hline $\begin{array}{llllllllll}\text { Mineral rico } & \text { Rammelsberg } & \ldots & \ldots & \ldots & \ldots & \ldots & \ldots & \ldots & \ldots\end{array}$ & 24,9 & 0,65 & 0,26 \\
\hline Mineral en betas Rammelsberg $\begin{array}{lllllll}\ldots & \ldots & \ldots & \ldots & \ldots & \ldots & \ldots\end{array}$ & 24,8 & 0,725 & 0,30 \\
\hline $\begin{array}{llllllllllll}\text { Mineral } & \mathrm{Pb}-\mathrm{Zn} \text { Lüderich } & \ldots & \ldots & \ldots & \ldots & \ldots & \ldots & \ldots & \ldots & \ldots & \ldots\end{array}$ & 22,2 & 0,79 & 0,395 \\
\hline $\begin{array}{lllllllllllll}\text { Mineral } \mathrm{Pb} & \text { Maubach } & \ldots & \ldots & \ldots & \ldots & \ldots & \ldots & \ldots & \ldots & \ldots & \ldots\end{array}$ & 17,9 & 0,87 & 0,71 \\
\hline Espato-flúor $\begin{array}{llllllllll} & \text { Finstergrund } \\
\ldots & \ldots & \ldots & \ldots & \ldots & \ldots & \ldots & \ldots & \ldots & \ldots\end{array}$ & 19,0 & 0,82 & 0,56 \\
\hline $\begin{array}{lllllllllllll}\text { Piedra caliza } & \text { Iberg } & \ldots & \ldots & \ldots & \ldots & \ldots & \ldots & \ldots & \ldots & \ldots & \ldots & \ldots\end{array}$ & 25,6 & 0,675 & 0,27 \\
\hline $\begin{array}{llllllllllll}\text { Grauwaca } \text { de } \operatorname{Harz} & \ldots & \ldots & \ldots & \ldots & \ldots & \ldots & \ldots & \ldots & \ldots & \ldots & \ldots\end{array}$ & 19,1 & 0,84 & 0,57 \\
\hline
\end{tabular}

\subsection{Resultados de los ensayos de Bond}

Los resultados de los índices de trabajo Wi hallados con arreglo a estas relaciones - convertidos en $\mathrm{kWh} / \mathrm{t}$ - están resumidos en la tabla 2 .

Comparando ambos valores de $\mathrm{Wi}_{\mathrm{B}}$, que se calcularon según la ecuación [8] ó [9], se manifiesta una concordancia relativamente buena - a excepción del mineral de betas de Rammelsberg-; las divergencias de los valores individuales son como máximo de \pm 10 $\%$. Es decir, que con una determinación aproximada del índice de trabajo $\mathrm{Wi}_{\mathrm{B}}$ es posible renunciar a la realización de los análisis granulométricos de alimentación y producto molido después de alcanzar el estado homogéneo de molienda, ya que las magnitudes $\mathrm{F}$ y $\mathrm{P}$ ya no entran en la ecuación [9].

Pero los resultados muestran claramente que el índice de trabajo $\mathrm{Wi}_{\mathrm{B}}$-tal como lo define Bond - sólo muy condicionadamente puede considerarse como una constante real del producto. Independientemente de la clase de mineral o roca analizados, se altera más o menos intensamente con la finura final $\mathrm{P}_{i}$ obtenida en el análisis de la aptitud a la molienda. El emplear el valor medio que resulta del índice de trabajo para diferentes finuras de molienda para el cálculo del consumo energético real necesario para la trituración con un determinado tamaño de grano de partida y finuras finales discrecionales, es, por lo tanto, sólo admisible para estimaciones muy aproximadas. Para un cálculo exacto hay que determinar el índice de trabajo $\mathrm{Wi}_{\mathrm{B}}$ para el tamaño de grano del producto molido que corresponda a la finura final planeada en la molienda industrial. En general, se recomienda realizar el ensayo de Bond para diversas finuras de molienda, ya que así se consigue una visión más clara de la magnitud de los valores del consumo energético en función del tamaño del producto molido.

La comparación de valores de los índices de trabajo $\mathrm{Wi}_{\mathrm{B}}$, según Bond, $\mathrm{y} \mathrm{Wi}_{\mathrm{H}}$, según Holmes, muestran las diferencias en la concepción de ambos autores. El índice de trabajo, según Holmes, permanece por definición casi constante con diferentes tamaños de grano de referencia; sólo el exponente $\mathrm{r}$ diverge más o menos del número 0,5. La escala de esta divergencia del valor de $\mathrm{r}$ hacia arriba y hacia abajo concuerda con un aumento o con una reducción más o menos intensos'de los índices de trabajo de Bond para diferentes finuras de molienda. 


\section{COMPARACION DE LOS VALORES DE CONSUMO DE ENERGIA OBTENIDOS EN LOS ENSAYOS DE LABORATORIO CON EL CONSUMO DE ENERGIA ESPECI- FICO EFECTIVO DE LOS MOLINOS INDUSTRIALES}

De los índices de trabajo determinados por el ensayo de laboratorio se calculó con ayuda de las relaciones básicas de Bond

$$
\mathrm{W}_{\mathrm{B}}=\mathrm{Wi}_{\mathrm{B}}\left(\frac{10}{\sqrt{\mathrm{P}}}-\frac{10}{\sqrt{\mathrm{F}}}\right)
$$

y de Holmes

$$
\mathrm{W}_{\mathrm{H}}=\mathrm{Wi}_{\mathrm{H}}\left(1-\frac{1}{\mathrm{R}^{\mathrm{r}}}\right)\left(\frac{100}{\mathrm{P}}\right)^{\mathrm{r}},
$$

el consumo específico de energía (en $\mathrm{kWh} / \mathrm{t}$ ) para la molienda industrial de los minerales analizados y se comparó con los valores obtenidos, por término medio, durante un tiempo prolongado en las respectivas instalaciones de preparación.

Los valores de $D_{80}$ de alimentación y excedente de clasificación se determinaron por la documentación de explotación de las instalaciones de preparación de Rammelsberg, Lüderich, Maubach y Finstergrund. Como índice de trabajo Wi se incluyó en el cálculo, por un lado, el valor obtenido en el ensayo de laboratorio para la trituración del mineral determinado a la finura de molienda correspondiente a las condiciones de la explotación (tabla 4, columnas 5 y 7); por el otro lado, se puso como base del índice de trabajo el valor medio de las cinco determinaciones (columnas 6 y 8 ).

T A B L A 4

Consumo específico de energía calculado y medido para la molienda

\begin{tabular}{|c|c|c|c|c|c|c|c|c|c|}
\hline \multirow{2}{*}{ M i n e r a 1} & \multirow{2}{*}{$\begin{array}{c}\text { Diámetro } \\
\text { del } \\
\text { molino } \\
\text { industrial } \\
\text { D } \\
(\mathrm{mm})\end{array}$} & \multirow{2}{*}{\begin{tabular}{|} 
Tamaño \\
de grano \\
de la ali- \\
mentación \\
F \\
$(\mu)$
\end{tabular}} & \multirow{2}{*}{\begin{tabular}{|l|} 
Tamaño \\
de grano \\
del pro- \\
ducto \\
molturado \\
$\quad$ P \\
$(\mu)$
\end{tabular}} & \multicolumn{2}{|c|}{$\begin{array}{l}\text { Consumo específi- } \\
\text { co de energía se- } \\
\text { gún Bond } W_{B} \\
(k W h / t)\end{array}$} & \multicolumn{2}{|c|}{$\begin{array}{l}\text { Consumo específi- } \\
\text { co de energía se- } \\
\text { gún Holmes } W_{\mathrm{H}} \\
(\mathrm{kWh} / \mathrm{t})\end{array}$} & \multirow{2}{*}{\begin{tabular}{|l} 
Media de \\
los valores \\
$\mathbf{W}_{\mathrm{H}} \mathbf{y} \mathbf{W}_{\mathrm{B}}$ \\
a igual fi- \\
nura de \\
molienda \\
$(\mathrm{kWh} / \mathrm{t})$
\end{tabular}} & \multirow{2}{*}{$\begin{array}{l}\text { Consumo } \\
\text { específico } \\
\text { de energía } \\
\text { del molino } \\
\text { industrial } \\
(\mathrm{kWh} / \mathrm{t})\end{array}$} \\
\hline & & & & $\begin{array}{l}\text { Finura de } \\
\text { molienda } \\
\quad \text { igual }\end{array}$ & $\begin{array}{l}\text { Valor } \\
\text { medio }\end{array}$ & $\begin{array}{l}\text { Finura de } \\
\text { molienda } \\
\text { igual }\end{array}$ & $\begin{array}{l}\text { Valor } \\
\text { medio }\end{array}$ & & \\
\hline (1) & (2) & (3) & (4) & (5) & (6) & $(7)$ & (8) & (9) & (10) \\
\hline $\begin{array}{l}\text { Mineral rico } \\
\text { Rammelsberg }\end{array}$ & 1.700 & 16.000 & 91 & 10,65 & 12,30 & 11,40 & 10,95 & 11,05 & 10,80 \\
\hline $\begin{array}{l}\text { Mineral } \\
\text { en betas } \\
\text { Rammelsberg }\end{array}$ & 2.100 & 1.280 & 145 & 7,75 & 8,15 & 6,60 & 6,90 & 7,20 & 7,30 \\
\hline $\begin{array}{l}\text { Mineral } \mathrm{Pb}-\mathrm{Zn} \\
\text { Lüderich }\end{array}$ & 1.700 & 17.800 & 137 & 10,50 & 11,20 & 10,25 & 10,60 & 10,40 & 10,00 \\
\hline $\begin{array}{l}\text { Mineral Pb } \\
\text { Maubach }\end{array}$ & 2.300 & 1.280 & 415 & 3,20 & 4,05 & 3,00 & 3,05 & 3,10 & 3,90 \\
\hline $\begin{array}{l}\text { Espato-flúor } \\
\text { Finstergrund }\end{array}$ & 1.500 & 12.400 & 200 & 8,65 & 8,00 & 6,75 & 6,75 & 7,70 & 7,40 \\
\hline
\end{tabular}
industrial de los minerales analizados 
Como ya se ha mencionado, el índice de trabajo Wi sólo es válido con exactitud para la molienda circulante húmeda en un molino de bolas de $2,45 \times 2,45 \mathrm{~m}$ cuyas condiciones de servicio han sido determinadas con precisión. Como quiera que los diámetros de los molinos industriales analizados divergen de esta cifra, en el cálculo del consumo energético específico se procedió también a la rectificación de los diámetros propuesta por Bond, (12):

$$
\mathrm{Wi}_{\mathrm{D}}=\mathrm{Wi}\left(\frac{2,45 \mathrm{~m}}{\mathrm{D}}\right)^{0,2} .
$$

Para el mineral de Maubach hubo que hacer todavía otra rectificación, ya que en esta instalación de preparación se muele el mineral en circuito abierto. Según Bond, (13), se supuso para esto:

$$
\mathrm{Wi}_{\text {abierto }}=1,4 \mathrm{Wi}_{\text {cerrado }} \text {. }
$$

Por la tabla 4 puede observarse que con el ensayo de Bond es posible una estimación relativamente exacta del consumo energético para la molturación de un material. Las diferencias entre consumo de energía calculado y medido son, a base del índice de trabajo $\mathrm{Wi}_{\mathrm{B}}$ determinado según Bond, como máximo, de $\pm 20 \%$. Aplicando el método de valoración propuesto por Holmes, dichas diferencias se reducen alrededor de $10 \%$ si no se tiene en cuenta la molienda del mineral de Maubach en circuito abierto. Los valores numéricos, calculados según Holmes, son más bajos que los números, determinados según Bond, los cuales, considerando la rectificación del diámetro, son siempre mayores que los valores de consumo determinados en las instalaciones de preparación.

En general, los valores calculados para el consumo energético, en los cuales se incluyó el índice de trabajo correspondiente a la finura del excedente de clasificación, concuerdan mejor con los datos de la explotación que los números calculados por los valores medios. El valor medio de los valores específicos de consumo energético, determinados según Bond y Holmes, para los tamaños de grano del producto molido en los minerales elegidos (columna 9), diverge sólo un poco del valor de la explotación (máximo 0,40 kWh/t, a excepción del mineral de Maubach). Con arreglo a estos resultados parece conveniente, al determinar el consumo energético específico para la molturación de minerales y rocas por medio del ensayo de Bond, efectuar la valoración tanto según Bond como según Holmes. Se recomienda aplicar el ensayo de Bond para diferentes finuras finales del producto a moler, con objeto de obtener un conocimiento más exacto del comportamiento a la aptitud de molienda y de la molturabilidad del material en función del tamaño de grano.

Los ensayos para este trabajo se realizaron en el Centro de Ensayos Humboldt, de la Klöckner-Humboldt-Deutz AG en Colonia, en el marco de un trabajo de investigación. Por el apoyo prestado durante los análisis se les da las gracias a los señores de esta firma que participaron, en especial al Ing. Dr. Wenz.

Relación de los símbolos empleados para la valoración de los ensayos de Bond

$\mathrm{A}=$ Parámetro en función del material.

$\mathrm{F}=$ Valor $\mathrm{D}_{80}$ de la alimentación $(\mu)$.

$\mathrm{G}=$ Coeficiente de aptitud a la molienda $(\mathrm{g} / \mathrm{r})$.

$\mathrm{P}=$ Valor de $\mathrm{D}_{80}$ del producto molido $(\mu)$.

$P_{\mathrm{i}} \quad=$ Finura de molienda del ensayo de Bond $(\mu)$. 


$$
\begin{aligned}
& \mathrm{q} \quad=\text { Parámetro en función del material. } \\
& \mathrm{r} \quad=\text { Exponente de trituración según Holmes. } \\
& \mathrm{R}=\text { Relación de trituración. } \\
& \mathrm{W}=\text { trabajo necesario total }(\mathrm{kWh} / \mathrm{t}) . \\
& \mathrm{W}_{\mathrm{B}}=\text { Trabajo necesario total calculado según Bond }(\mathrm{kWh} / \mathrm{t}) . \\
& \mathrm{W}_{\mathrm{H}}=\text { trabajo necesario total calculado según Holmes }(\mathrm{kWh} / \mathrm{t}) . \\
& \mathrm{Wi}=\text { Indice de trabajo }(\mathrm{kWh} / \mathrm{t}) . \\
& \mathrm{Wi}_{\mathrm{B}}=\text { Indice de trabajo según Bond }(\mathrm{kWh} / \mathrm{t}) . \\
& \mathrm{Wi}_{\mathrm{H}}=\text { Indice de trabajo según Holmes }(\mathrm{kWh} / \mathrm{t}) .
\end{aligned}
$$

\section{B I B L I O G R A F I A}

(1) Rose, H. E.: Eine Untersuchung des Kraftbedarfs von Kugelmühien unter Verwendung von Kleinmodellen. TIZ-Zbl. 80 (1956), S. 143-153.

(2) B o in d, F. C.: The third theory of comminution. Transact. AIME 193 (1952), S. 484-494.

(3) Holmes, J. A.: A contribution to the study of comminution - A modified form of Kick's law. Transact. Instn. Chem. Engrs. 35 (1957), S. 12j-156.

(4) B o n d, F. C.: Standard grindability tests tabulated. Transact. AIME 183 (1949), S. 313-329.

(5) Perow - Bran d : Feinmahlen der Erze. Berlin (1954), S. 17-25.

(6) Mittag, C.: Prüfverfahren zur Ermittlung von Höchstleistungen in Kugel- und Rohrmühlen. Berlin, Göttingen, Heidelberg (1954), $41 \mathrm{~S}$.

(7) Denver Equipment Company: Grinding mills. Bulletin Nr. B $2-$ B 34, S. $50-51$.

(8) ASTM Standard D $409-51$ : Standard method of test for grindability of coal by the Hardgrove-machine method. ASTM Standards on coal and coke. American Society for Testing Materials. Philadelphia (1959), September, S. $49-52$.

(9) L e hma $\mathrm{n} n$, H., und $\mathrm{H}$ a e s e, V.: Der Mahlbarkeitsprüfer, ein Gerät zur Untersuchung der Mahlbarkeit harter Stoffe. TIZ-Zbl. 79 (1955), S. 91-94.

(10) Warren Spring Laboratory: Grindability test procedure and bail-mill size selection. Mincral Processing Note Nr. 3 (1962), $18 \mathrm{~S}$.

(11) B o n a, F. C.: Confirmation of the third theory. Transact. Arive 217 (1960), S. 139-153.

(12) B o n d, F. C.: New equation for calculating the work-index from A-C closed circuit ball mill grindability tests. Allis Chalmers Publication, 1960.

(13) B o n d, F. C.: Berechnungsmethode zur Feinzerkleinerung. Aufbereitungs-Technik 5 (1964), S. 211-218. 